infarct size was similar (38.3 $\pm 2.0 \%$ vs $39.9 \pm 2.8 \%$ ). Transgenic mice also exhibited less apoptosis and interstitial cardiac fibrosis $(0.52 \pm 0.03 \%$ vs $0.65 \pm 0.05 \%, p<0.05)$, and lower increases in the expression of fibronectin and procollagen III mRNA. Both echocardiography and cardiac catheterisation demonstrated less left ventricular cavity dilatation and a preservation of cardiac function in transgenic than wt mice.

Conclusions In contrast to Nox2, which contributes to cardiac dilation, contractile dysfunction and fibrosis, cardiomyocyte Nox4 was found to exert protective effects against adverse remodelling post-MI

\section{A NEW MOLECULAR MECHANISM FOR FAMILIAL DILATED CARDIOMYOPATHY BASED ON STUDIES WITH INTACT MUTANT TRANSGENIC MOUSE AND HUMAN EXPLANTED HEART MUSCLE}

doi:10.1136/hrt.2009.191049k

M Memo, A E Messer, W Song, S B Marston. Imperial College, London, UK

We created a transgenic mouse that expressed the dilated cardiomyopathy (DCM) mutation ACTC E361G at 50\% of total cardiac actin. We isolated F-actin from transgenic and non-transgenic (NTG) mice and reconstituted thin filaments using native human cardiac troponin and tropomyosin (from donor heart). In in-vitro motility assays we could observe no differences between E361G and nontransgenic mouse thin filaments; however, when troponin was fully dephosphorylated with acid phosphatase we observed that E361G $\mathrm{Ca}^{2+}$ sensitivity was lower than non-transgenic, as previously observed with the recombinant proteins (EC50 E361G/NTG $2.2 \pm 0.1)$. When we compared natively phosphorylated and dephosphorylated thin filaments we observed that $\mathrm{Ca}^{2+}$ sensitivity did not change in E361G mouse thin filaments (EC ${ }_{50} \mathrm{P} /$ unP $=1.0 \pm 0.1$ ) but the $\mathrm{Ca}^{2+}$ sensitivity increased $3.0 \pm 0.3$-fold on dephosphorylation of non-transgenic mice as expected. Thus the only functional change induced by the E361G mutation in cardiac actin was a blunted response to troponin I phosphorylation. We also studied troponin extracted from the explanted heart of a DCM patient carrying the cardiac TnC G159D mutation. In-vitro motility assay investigation of reconstituted thin filaments showed that the cTnC G159D mutation also showed little change in $\mathrm{Ca}^{2+}$ sensitivity when TnI was dephosphorylated $\left(E_{50} \mathrm{P} / \mathrm{unP}=1.2 \pm 0.2\right)$. In contrast, with donor heart control troponin, $\mathrm{Ca}^{2+}$ sensitivity was increased $\left(\mathrm{EC}_{50} \mathrm{P} / \mathrm{unP}=4.7 \pm 1.9\right)$. We conclude that $\mathrm{Ca}^{2+}$ sensitivity per se is not the prime determinant of familial DCM. The causative property shared by mutations in contractile proteins that cause DCM is a blunted response to changes in troponin I phosphorylation that could impair the normal response to adrenergic stimulation.

Funding This research was supported by grants from the British Heart Foundation

\section{IS NITRIC OXIDE SYNTHASE PRESENT IN MITOCHONDRIA?}

doi:10.1136/hrt.2009.191049|

W H Y Cheng, S M Carter, A P Halestrap, E J Griffiths. Department of Biochemistry and Bristol Heart Institute, University of Bristol, Bristol, UK

In addition to the three known forms of nitric oxide synthase (NOS) in the heart, it has recently been proposed that NOS is also present in mitochondria. However, studies are controversial due to the possibility of contamination by non-mitochondrial NOS, and because none of the known forms of NOS contain a mitochondrial targeting sequence. We investigated whether NOS was present in isolated mitochondria using antibodies against all three forms of NOS (endothelial NOS (eNOS), inducible NOS (iNOS) and neuronal NOS (nNOS)). Crude fractions of heart and liver mito- chondria were obtained by differential centrifugation, and $35 \%$ Percoll was used to obtain highly purified mitochondria, as tested using antibodies against subcellular marker proteins: cyclophilin $\mathrm{D}$, mitochondrial marker; monocarboxylate transporter-1, plasma membrane marker; ryanodine receptor, sarcoplasmic reticulum marker (heart mitochondria only) and catalase, peroxisomal marker (liver only). Western blotting using antibodies against eNOS and iNOS revealed that these isoforms were not present in either heart or liver purified mitochondria (whereas whole heart or liver lysate tested positive). We used five different antibodies against nNOS, and again failed to detect anything in purified heart mitochondria However, in purified liver mitochondria one of the nNOS antibodies revealed the presence of a band at the correct molecular weight. We are currently determining whether this is indeed nNOS. In addition, we will assay for NOS activity in the purified mitochondria. Nitric oxide can inhibit mitochondrial respiration, so the existence of mitochondrial NOS may provide an important modulatory mechanism for respiration under either physiological or pathological conditions.

Funding This study was supported by the BBSRC and NiCox.

\section{REGULATION OF GENE TRANSCRIPTION BY HYDROGEN PEROXIDE IN CARDIOMYOCYTES}

doi:10.1136/hrt.2009.191064a

K Lay, A K Marshall, E Amirak, 0 Barrett, E L Tham, T Markou, S J Fuller, P H Sugden, A Clerk. National Heart and Lung Institute, Cardiovascular Science, Imperial College London, London SW7 2AZ, UK

Introduction Reactive oxygen species (ROS) levels rise during ischaemia and reperfusion and may contribute to myocardial injury, remodelling and progression to heart failure. ROS (hydrogen peroxide; $\mathrm{H}_{2} \mathrm{O}_{2}$ ) activates both apoptotic and pro-survival signalling pathways, although at higher concentrations cardiomyocyte death invariably results. Here, transcriptional responses of cardiomyocytes to varying concentrations and duration of $\mathrm{H}_{2} \mathrm{O}_{2}$ were investigated.

Methods Neonatal rat ventricular myocytes were exposed to 0.05 $1.0 \mathrm{mM} \mathrm{H}_{2} \mathrm{O}_{2}$ for up to $6 \mathrm{~h}$. Messenger RNA expression of selected genes was analysed by quantitative PCR. Cycloheximide was used to define immediate early genes (IEG) and second-phase genes.

Results Transcription factors of activating protein 1 (AP-1) and early growth response (EGR) families were upregulated rapidly and transiently by $0.1-1 \mathrm{mM} \mathrm{H}_{2} \mathrm{O}_{2}$, concentrations, which induce cardiomyocyte apoptosis and are IEG. In contrast, upregulation of transcripts for antioxidant proteins (second-phase genes) was slower and more sustained, occurring at a lower concentration of $\mathrm{H}_{2} \mathrm{O}_{2}(0.05-0.5 \mathrm{mM})$. Conclusions AP-1, EGR and antioxidant transcripts were regulated by $\mathrm{H}_{2} \mathrm{O}_{2}$ in a time and concentration-dependent manner. Induction of antioxidant expression at lower concentrations of $\mathrm{H}_{2} \mathrm{O}_{2}$ could represent enhancement of ROS-scavenging capacity to prevent apoptosis At higher $\mathrm{H}_{2} \mathrm{O}_{2}$ concentrations, the AP-1 and EGR transcription factor IEG may mediate apoptosis. These studies increase the understanding of transcriptional responses in cardiomyocytes to ROS.

\section{THIN FILAMENTS RECONSTITUTED WITH TROPONIN EXTRACTED FROM PATIENTS WITH HYPERTROPHIC OBSTRUCTIVE CARDIOMYOPATHY ARE FUNCTIONALLY ABNORMAL}

doi:10.1136/hrt.2009.191064b

C R Bayliss, A E Messer, O Copeland, C E Gallon, S B Marston. Imperial College, London, UK

Tissue obtained from a septal myectomy represented an opportunity to characterise the molecular phenotype of hypertrophic 
obstructive cardiomyopathy (HOCM). We have isolated troponin from HOCM muscle and studied its function using the in-vitro motility assay (IVMA). The level of troponin I (TnI) phosphorylation has been measured as $0.29 \pm 0.04 \mathrm{~mol} \mathrm{Pi} / \mathrm{mol}$ TnI compared with $1.62 \pm 0.06 \mathrm{~mol} \mathrm{Pi} / \mathrm{mol} \mathrm{TnI}$ in donor heart samples. The $\mathrm{Ca}^{2+}$-sensitivity of reconstituted thin filaments containing donor and HOCM troponin were measured. The HOCM troponin conferred a $\mathrm{Ca}^{2+}$-sensitivity that was not significantly different to that of the donor troponin. $\mathrm{EC}_{50} \mathrm{HOCM} /$ donor $=0.88 \pm 0.22(\mathrm{n}=8)$. HOCM troponin was treated with protein kinase A (PKA) (catalytic subunit) to increase the level of TnI phosphorylation to a similar level as found in donor hearts. There was no significant difference in the $\mathrm{Ca}^{2+}$ sensitivities of the thin filaments reconstituted with untreated or PKA-treated HOCM troponin. EC 50 HOCM/PKA treated HOCM=0.93 $\pm 0.32(n=4)$. Finally, donor troponin was dephosphorylated by treatment with acid phosphatase. In preliminary experiments this was compared with HOCM troponin, which has an intrinsically low level of phosphorylation. Thin filaments reconstituted with dephosphorylated donor troponin had a higher $\mathrm{Ca}^{2+}$ sensitivity than thin filaments containing HOCM troponin. Therefore, we conclude that HOCM troponin is modified in a manner independent of the causative mutation. HOCM troponin confers a $\mathrm{Ca}^{2+}$ sensitivity similar to troponin from donor hearts, which is independent of the phosphorylation status of TnI. This molecular phenotype is different from both acquired heart failure and genetic dilated cardiomyopathy.

\section{INVESTIGATING METABOLIC FLUX IN THE HYPERTHYROID HEART USING HYPERPOLARISED MAGNETIC RESONANCE}

doi:10.1136/hrt.2009.191064c

M Dodd, H Atherton, M Schroeder, L Heather, J Griffin, K Clarke, G Radda, D Tyler. Cardiac Metabolism Research Group, Department of Physiology, Anatomy and Genetics, University of Oxford, Oxford, UK

An increase in circulating thyroid hormone (TH) causes hypertrophy, which is accompanied by an increase in contractility and cardiac output. However, the mechanisms behind these observations are yet to be fully elucidated. TH is an important regulator of energy metabolism and has been shown to control the expression of many important lipolytic and glycolytic enzymes. One such enzyme is pyruvate dehydrogenase kinase, which, via phosphorylation, reduces the activity of pyruvate dehydrogenase $(\mathrm{PDH})$ and thus decreases the conversion of pyruvate to acetyl CoA, a key substrate in ATP synthesis. However, it is not known whether PDH activity is inhibited in the hyperthyroid rat heart. Hyperpolarised substrates increase the sensitivity of magnetic resonance spectroscopy (MRS) so that it is possible to investigate the flux of metabolites through specific enzymes in vivo. In this study, hyperpolarised pyruvate has been used in conjunction with MRS localised to the heart to monitor real time metabolic flux through $\mathrm{PDH}$ in hearts of control rats and rats injected with $\mathrm{TH}$ (triiodothyronine; T3) for 7 days ( $n=8$ per group). PDH activity measurements were made at baseline and after 7 days of injections. Hyperpolarised pyruvate was injected over $10 \mathrm{~s}$ into the anaesthetised rat via the tail vein. The conversion of pyruvate to alanine, lactate and bicarbonate was monitored every $1 \mathrm{~s}$ for 1 minute. The bicarbonate/pyruvate ratio was used as a measure of flux through $\mathrm{PDH}$. After 7 days administration of T3, flux through cardiac PDH was reduced by $76 \%(p<0.01)$. Thus, hyperpolarised pyruvate has revealed $\mathrm{PDH}$ inhibition to contribute to the pathology of the hyperthyroid heart.

\section{EVIDENCE FOR INWARD RECTIFIER POTASSIUM CHANNELS IN HL-1 CELLS}

doi:10.1136/hrt.2009.191064d

D Goldoni, A Collins. Centre for Vision and Vascular Science, School of Medicine and Dentistry, Queens University Belfast, Belfast, UK

Cardiac inward rectifier potassium current (IK1) plays a paramount role in repolarisation and stabilisation of the membrane potential in cardiomyocytes. Its downregulation contributes to arrhythmogenesis in heart failure and post-myocardial infarction. We have undertaken the molecular and functional characterisation of IK1 in the HL-1 cell line, which is derived from a mouse atrial tumour. HL-1 cells express many genes typical of differentiated adult cardiomyocytes and so far they are the only cardiac cell line able to divide continuously while maintaining a differentiated cardiac phenotype. ${ }^{1}$ IK1 has not been described in HL-1 cells. Whole-cell recording was performed under conditions that have been shown to preserve IK1 in excised membrane patches; the pipette solution contained $10 \mathrm{mM}$ pyrophosphate, $5 \mathrm{mM}$ fluoride and $0.1 \mathrm{mM}$ vanadate. Extracellular barium at an IK1-selective concentration $(0.1 \mathrm{mM}$ ) inhibited inward current by $44 \% \pm 4.2 \%$ (mean \pm SEM, $n=13$ cells), with little or no effect on outward current. The apparent reversal potential of the barium-sensitive current was $-58 \pm 6.0 \mathrm{mV}$ in $20 \mathrm{mM}$ and $-10 \pm 3.9 \mathrm{mV}$ in $100 \mathrm{mM}$ extracellular potassium with $160 \mathrm{mM}$ potassium in the pipette $(n=7)$. Reverse transcriptase PCR was performed with HL-1 complimentary DNA and primers specific for Kir2.1 (KCNJ2), the dominant IK1 isoform in cardiomyocytes. A PCR fragment of the expected size and sequence was produced. These results support the conclusion that HL-1 cells have an inwardly rectifying potassium current and express Kir2.1.

Acknowledgements The authors would like to thank Professor Barbara J McDermott for HL-1 cells and Dr You You Zhao for HL-1 cDNA.

Funding D Goldoni is supported by a studentship from the Harold McCauley Fund for Cardiovascular Research.

\section{REFERENCE}

1. Claycomb, et al. Proc Natl Acad Sci U S A 1998;95:2979-84.

\section{CONCENTRATION-DEPENDENT PROTECTION BY NO DONORS AGAINST ISCHAEMIA-REPERFUSION INJURY IN PERFUSED RAT HEARTS}

doi:10.1136/hrt.2009.191064e

W H Y Cheng, A P Halestrap, E J Griffiths. Department of Biochemistry and Bristol Heart Institute, University of Bristol, Bristol, UK

Nitric oxide (NO) has the potential to be beneficial in attenuating ischaemia-reperfusion (IR) injury, or deleterious due to its tendency to form reactive nitrogen species (RNS). In addition, different donors release varying amounts of $\mathrm{NO}$, making them difficult to compare directly. We studied the effects of two NO donors, SNAP (S-nitroso- $N$-acetyl-DL-penicillamine) and DEA/NO (diethylamine NONOate). Isolated rat hearts were perfused with or without SNAP at $2-100 \mu \mathrm{M}$ or DEA/NO at $2-20 \mu \mathrm{M}$. The NO donors were added 20 minutes before the onset of ischaemia ( 30 minutes) and remained present throughout reperfusion (60 minutes). Injury was assessed by measuring left ventricular developed pressure (LVDP) and lactate dehydrogenase $(\mathrm{LDH})$ release. SNAP significantly protected hearts from IR injury at 20 and $40 \mu \mathrm{M}$, but not $2 \mu \mathrm{M}$ or $100 \mu \mathrm{M}$, compared with controls, as indicated by improved recovery of LVDP and reduced $\mathrm{LDH}$ release ( $\mathrm{n}=6$ for each condition). Protection with DEA/ $\mathrm{NO}$ was seen at $2 \mu \mathrm{M}$ but at $20 \mu \mathrm{M}$ protection was lost. In conclusion, NO donors protected hearts, but the effect was concentration dependent; protection being lost at higher levels of the 